\title{
Tipo de parto y sensibilidad materna: Efectos en el desarrollo socioemocional de niños y niñas al año de edad
}

\author{
Rodrigo Ramos; Verónica Yávar; Alejandro Del Río; Javiera Schettino; Valentina Bresciani; \\ Diana Gómez; Carolina Álvarez; Chamarrita Farkas
}

How to cite this article:

Ramos, R., Yávar, V., Del Río, A., Schettino, J., Bresciani, V., Gómez, D., Álvarez, C., \& Farkas, Ch. (2020). Mode of Delivery and Maternal Sensitivity: Effects on the Socioemotional Development of Children at One Year of Age. Acta Colombiana de Psicología, 23(2), 241-253. http://www.doi.org/10.14718/ACP.2020.23.2.10

Recibido, septiembre 02/2019; Concepto de evaluación, diciembre 09/2019; Aceptado febrero 18/2020

\author{
Rodrigo Ramos \\ Pontificia Universidad Católica de Chile, Santiago, Chile \\ ORCID: https://orcid.org/0000-0003-0723-6713 \\ Verónica Yávar \\ Pontificia Universidad Católica de Chile, Santiago, Chile \\ ORCID: https://orcid.org/0000-0002-5700-7647 \\ Alejandro Del Río \\ Pontificia Universidad Católica de Chile, Santiago, Chile \\ ORCID: https://orcid.org/0000-0001-6496-9601 \\ Javiera Schettino \\ Pontificia Universidad Católica de Chile, Santiago, Chile \\ ORCID: https://orcid.org/0000-0002-6793-9317 \\ Valentina Bresciani \\ Pontificia Universidad Católica de Chile, Santiago, Chile \\ ORCID: https://orcid.org/0000-0003-2465-1943 \\ Diana Gómez \\ Pontificia Universidad Católica de Chile, Santiago, Chile \\ ORCID: https://orcid.org/0000-0001-8507-6714 \\ Carolina Álvarez \\ Pontificia Universidad Católica de Chile, Santiago, Chile \\ ORCID: https://orcid.org/0000-0001-8287-7751 \\ Chamarrita Farkas* \\ Pontificia Universidad Católica de Chile, Santiago, Chile \\ ORCID: https://orcid.org/0000-0002-0438-9354
}

\begin{abstract}
Resumen
La infancia temprana es un periodo de gran relevancia en el desarrollo socioemocional infantil; de hecho, es donde se sientan las bases del desarrollo futuro. En este sentido, las adquisiciones durante el primer año de vida son predictores importantes de las habilidades sociales y emocionales futuras, de modo que la sensibilidad materna, según evidencia respecto a sus efectos en el desarrollo socioemocional del niño, ha demostrado ser un aspecto fundamental durante este periodo. Teniendo esto en cuenta, y considerando la relevancia de dicha competencia, así como el alza mundial y nacional en las tasas de cesárea y los posibles riesgos asociados al tipo de parto vivenciado, el presente estudio tuvo como objetivo analizar la influencia de la sensibilidad materna y del tipo de parto en el desarrollo socioemocional infantil al año de edad. Para ello, se estudió una muestra no probabilística intencionada de 91 madres con sus respectivos hijos o hijas — de distinto nivel socioeconómicoque asistían a salas cuna públicas o privadas en la ciudad de Santiago, Chile, por medio de dos instrumentos: la escala de sensibilidad del adulto (ESA) y la functional emotional assessment scale (FEAS). En general, los resultados no mostraron diferencias en el desarrollo socioemocional de los niños según el tipo de parto, aunque sí se pudo apreciar una relación entre una mayor sensibilidad materna y un mayor desarrollo socioemocional de los niños al año de edad. Al final se discuten las implicaciones de promover la sensibilidad materna con el fin de apoyar un óptimo desarrollo socioemocional en los infantes. Palabras clave: tipo de parto, sensibilidad materna, desarrollo socioemocional, infancia temprana.
\end{abstract}

* Escuela de Psicología, Av. Vicuña Mackenna, 4860, Macul, Santiago, Chile. Tel.: 56-2-23547067. chfarkas@uc.cl Esta investigación fue financiada por el Fondo Nacional de Desarrollo Científico y Tecnológico (FONDECYT) n. ${ }^{\circ} 1160110$. 


\title{
Mode of Delivery and Maternal Sensitivity: Effects on the Socioemotional Development of Children at One Year of Age
}

\begin{abstract}
Early childhood is a period of high relevance in children's socioemotional development, establishing the basis for future development. Acquisitions during the first year of life are significant predictors of future social and emotional skills. During this period, maternal sensitivity is also essential, and there is evidence regarding its effects on the socioemotional development of the child. Considering the relevance of this competence, together with the global and national increase in cesarean rates and the possible risks associated with the type of delivery experienced, the influence of the type of delivery and the maternal sensitivity in child's socioemotional development at one year of age was analyzed. For this, an intentional non-probabilistic sample of 91 mothers with their respective children of different socioeconomic levels, who attended public or private nurseries in the city of Santiago, Chile, was studied. The instruments used were the Adult Sensitivity Scale (ESA) and the Functional Emotional Assessment Scale (FEAS). The results showed no differences in the children's socioemotional development according to the type of delivery. However, it was possible to observe an association between a higher maternal sensitivity and a more significant socioemotional development on children at one year of age. The implications of promoting maternal sensitivity are discussed to support optimal socioemotional development in infants.

Keywords: type of delivery, maternal sensitivity, socioemotional development, early infancy.
\end{abstract}

\section{Introducción}

Según lo reportado en la literatura científica, por desarrollo socioemocional se entiende el funcionamiento social y emocional del ser humano, en el cual se incluyen las capacidades de autorregulación, el apego, las claves emocionales y la señalización, entre otras funciones específicas (Greenspan et al., 2001). Sin embargo, estas capacidades deben pasar por un entorno de desarrollo adecuado, ya que para poder experimentar, regular y expresar emociones de una manera apropiada es necesario que el niño o niña (en adelante, "niño") cuente con un desarrollo individual apropiado y seguro frente a su cuidador o cuidadora (en adelante, "cuidador") (Palmer et al., 2013).

De hecho, uno de los momentos más importantes del desarrollo socioemocional es el que ocurre alrededor del año de edad, pues es en esta etapa donde aparecen la incorporación de deseos e intenciones al comportamiento, así como la integración de distintos sentidos y el reconocimiento de una gestualidad preverbal relacionada íntimamente con las emociones. Así, dada su importancia, un déficit en el desarrollo de esta etapa se ha relacionado tanto con problemas psicosociales como con fallas en el aprendizaje temprano (Greenspan et al., 2001).

En este sentido, el desarrollo socioemocional durante el primer año de vida se ha identificado como un importante predictor del desarrollo socioemocional de los niños, ya que, como se demuestra en diversos estudios, los niños que llegan con un desarrollo socioemocional adecuado a la edad escolar tienen mejores competencias académicas y logran un ajuste más adecuado, además de que se identifica en ellos una reducción del riesgo de problemas y de violencia escolar (Berger et al., 2009; Domitrovich et al., 2017). Por ello, lograr adecuadas competencias socioemocionales de manera temprana representa un aspecto fundamental tanto en el desarrollo humano como en la preparación de la vida (De Andrés, 2005).

Ahora bien, debido a la relevancia de las competencias socioemocionales en la primera infancia, el estudio de los predictores del desarrollo de estas competencias ha permitido dar cuenta de aspectos importantes no solo para intervenciones educativas, sino también para el desarrollo de políticas públicas; y en dichos estudios, dentro de los factores predictores del desarrollo socioemocional infantil destacan las características de los padres —como su autorregulación, temperamento y estado de salud - , la relación entre padres e hijos, la crianza y el ambiente familiar, debido a que contribuyen, tanto individualmente como en conjunto, al desarrollo de habilidades de autorregulación en el infante (Kiss et al., 2014).

Sin embargo, dentro de estos factores hay uno que destaca con gran relevancia, y es el relacionado con la cualidad de la madre en el vínculo afectivo con el infante, ya que es ella quien cumple una función imprescindible durante los primeros 12 meses de edad de cualquier ser humano (Simó \& D'Ocon, 2014), y porque durante el primer año de vida este vínculo es el que cobra mayor relevancia, sobre todo porque es a partir de este que se logra el desarrollo de habilidades autorregulatorias en el niño (Farkas et al., 2017).

Ahora bien, en el contexto chileno las competencias parentales, tales como la sensibilidad materna y la mentalización, así como el nivel socioeconómico familiar y el 
sexo del infante, también han demostrado ser predictores relevantes del desarrollo socioemocional del niño (Farkas et al., 2017); no obstante, hacen falta estudios con población chilena que aborden la relación entre estas competencias parentales - posibles de promover e intervenir tempranamente - y el desarrollo socioemocional del infante. En este sentido, resulta relevante definir claramente a lo que se refiere la sensibilidad materna y su relación con el desarrollo socioemocional infantil.

Primero, la sensibilidad materna se entiende como la capacidad de la madre de captar adecuadamente las señales y comunicaciones del niño, y responder a ellas de manera pronta y efectiva (Ainsworth et al., 1978; Santelices et al., 2012); definición que resulta coherente con la teoría del apego de Bowlby (1969), donde el elemento central para el desarrollo social, cognitivo y emocional del individuo es que el cuidador responda a las necesidades y conducta del infante de manera tal que este logre regular sus emociones y sentirse seguro (Bordoni, 2018; Morera et al., 2012), mientras, a la vez, desarrolla su capacidad de adaptación (Bornstein \& Putnick, 2012).

De este modo, la calidad afectiva de la interacción temprana entre madre e hijo resulta de suma relevancia para el desarrollo cognitivo y socioemocional de este último, sobre todo porque dicha relación que permite predecir los índices de desarrollo cognitivo, psicomotor, lingüístico, académico y socioemocional en el niño (Kopystynska et al., 2016; Moed et al., 2017; Simó \& D’Ocon, 2014). De igual forma, en la literatura científica se encuentra que el desarrollo de adecuadas habilidades socioemocionales durante el primer año de vida le permite al infante organizar y construir un esquema de trabajo interno para representar la realidad de la relación y generar expectativas sobre cómo comportarse en la interacción con el adulto, y así obtener mayor nivel de protección (Atkinson et al., 2000).

Adicional a esto, la sensibilidad materna también se ha entendido como una competencia altamente relacionada con variables contextuales, como lo son el nivel socioeconómico (NSE) de la familia (Bornstein et al., 2007), el ingreso familiar (Pelchat et al., 2003) y el nivel educacional de la madre (Doesum et al., 2007; Pelchat et al., 2003). En Chile, por ejemplo, se ha podido constatar que niveles socioeconómicos, educacionales u ocupacionales más altos se asocian a una mayor probabilidad de que la madre presente una sensibilidad más alta hacia el niño cuando este tiene un año de edad (Santelices et al., 2015). Asimismo, madres provenientes de un NSE bajo presentan una disminución en su comportamiento sensible, es decir, en la disponibilidad y calidad de respuesta hacia las necesidades del niño (Gálvez \& Farkas, 2017).
De este modo, considerando que para el año 2017 en Chile un $20.7 \%$ de la población se ubicó en una situación de pobreza multidimensional (Ministerio de Desarrollo Social, 2018), analizar la relación entre la sensibilidad materna y el desarrollo socioemocional de sus hijos a temprana edad se convierte en un aspecto a evaluar de cada vez mayor importancia, sobre todo por sus implicaciones para el desarrollo de políticas públicas.

Por otra parte, a diferencia de los aspectos contextuales descritos anteriormente, pocos estudios han abordado si aspectos vinculados al embarazo y al parto podrían afectar la sensibilidad materna y, por ende, el desarrollo socioemocional de los niños - aunque los estudios en depresión pre y postparto son una excepción- (véase, por ejemplo, Field, 2010; Kemppinen et al., 2006). Sin embargo, dentro de los escasos estudios en este tema se ha observado que madres que no experimentaron problemas de salud durante su embarazo, cuyos embarazos fueron de mayor duración, y que tuvieron partos vaginales e hijos con un mayor peso o edad gestacional al nacer manifestaban, luego, una mayor sensibilidad materna hacia sus hijos (Bernier et al., 2010; Shin et al., 2006). Teniendo esto último en cuenta, llama la atención la variable de el tipo de parto, debido a que durante los últimos años ha habido en Chile un preocupante incremento de la tasa de nacimientos por cesárea (Ministerio de Salud, 2015). De este modo, se reconoce también la importancia de indagar si el tipo de parto tiene algún impacto sobre el desarrollo socioemocional infantil.

\section{Tipo de parto, sensibilidad materna y desarrollo socioemo- cional infantil}

En las últimas décadas, el acelerado aumento de las tasas de parto por cesárea se ha vuelto una preocupación urgente a nivel mundial (Lee \& Kirkham, 2008); problema que para el año 2010 había alcanzando en Chile un $76 \%$ en el sistema privado y un $37 \%$ en el sistema público, y que para el 2013 había avanzando al $40.5 \%$ para el sistema público (Ministerio de Salud, 2015), con su consecuente disminución de los nacimientos por parto vaginal (Salinas et al., 2007). Adicional a esto, se encuentra que un $15 \%$ de los partos a nivel mundial corresponden a cesáreas (Betrán et al., 2007), y que la tasa de cesáreas recomendada por la OMS no debe superar el 10-15\% (WHO, 1985, citado por Salinas et al., 2007), lo cual sitúa a Chile como uno de los países con mayor cantidad de cesáreas en el mundo.

En general, el parto suele representar para las mujeres un evento en donde predominan sentimientos de miedo, dado que se ha transmitido históricamente un discurso que caracteriza a este momento como un evento "peligroso" y que "conlleva riesgos" (I Font, 2015). A la vez, se ha 
comprobado que la experiencia y expectativas de las mujeres con respecto a la manera en que viven el parto tienen una fuerte repercusión en ellas, pues se han encontrado diferencias entre aquellas que experimentan partos vaginales y quienes deben realizarse cesáreas programadas y no programadas. Así, por ejemplo, las mujeres que tienen una cesárea no programada presentan niveles de ansiedad significativamente mayores en comparación a aquellas que vivencian un parto vaginal (Ryding et al., 1998), a la vez que perciben un impacto negativo sobre sus competencias maternas y sobre el vínculo inicial con sus hijos (Van Reenen \& Van Rensburg, 2013). En este sentido, resulta preocupante el hecho de que se haya encontrado que una mayor ansiedad durante el embarazo afecte negativamente la salud gestacional, aumente el riesgo obstétrico y obstaculice una normal adaptación a la maternidad (Osório et al., 2018).

De igual forma, se encuentra que los distintos tipos de parto afectan las percepciones respecto al recién nacido y los comportamientos parentales de la madre (Lobel \& DeLuca, 2007), puesto que las mujeres que tienen parto por cesárea, en contraste a un parto vaginal, presentan un humor más depresivo, evalúan a su hijos de una manera menos favorable y les proveen menos estimulación, cuidados y juego en los primeros cinco meses postparto (Lobel \& DeLuca, 2007). Incluso, diversos estudios muestran una mayor tasa de depresión postparto en mujeres que han tenido un parto por cesárea, en comparación a aquellas que han tenido un parto vaginal (Xie et al., 2011); no obstante, no en todas las culturas el parto por cesárea se ha asociado a una mayor depresión postnatal (véase, p. ej., Goker et al., 2012; Sword et al., 2011).

En esta misma línea, Uriarte (2006) identifica a la cesárea como un factor de riesgo para la depresión postparto, estado que, a su vez, puede afectar incluso de manera permanente la formación de vínculos de apego, dado que la madre ve mermada su capacidad de vincularse emocionalmente con el recién nacido. Incluso se ha encontrado una asociación significativa entre el parto vaginal y una mejor calidad de apego entre la madre y su hijo (Lecannelier et al., 2008), lo cual puede, a su vez, promover un mayor desarrollo socioemocional en el niño.

Ahora bien, considerando las diferencias en las repercusiones que tiene cada tipo de parto (Lobel \& DeLuca, 2007; Peters et al., 2018; Ryding et al., 1998), así como las implicaciones de un parto por cesárea en la salud de la madre y del niño - como el incremento de problemas relacionados con la función pulmonar, alteraciones en el metabolismo y trastornos de presión sanguínea, entre otros- (Peters et al., 2018), resulta necesaria, por tanto, la realización de estudios que incorporen esta variable y sus posibles efectos sobre el desarrollo infantil.
$\mathrm{Al}$ respecto, cabe mencionar que no hay literatura que explicite una relación entre el tipo de parto y el desarrollo socioemocional del infante, a pesar de que algunos estudios sí muestren una relación entre tipo de parto y sensibilidad materna, donde se encuentra que cuando el parto es por cesárea las madres son menos sensibles hacia sus bebés que cuando el parto es de tipo vaginal (Bernier et al., 2010; Swain et al., 2008); efecto especialmente destacado en el caso de cesáreas no programadas, donde el impacto negativo en el vínculo con su hijo era mayor (Van Reenen \& Van Rensburg, 2013). Dichas condiciones podrían impactar indirectamente en el desarrollo socioemocional de los niños.

Finalmente, teniendo en cuenta los antecedentes descritos, el presente estudio tuvo como objetivo analizar la influencia del tipo de parto y de la sensibilidad materna sobre el desarrollo socioemocional de los infantes, ya que este último tiene una gran influencia tanto en las competencias académicas del niño, así como en su ajuste adecuado y en la reducción del riesgo de problemas y de violencia escolar, entre otros aspectos (Berger et al., 2009; Domitrovich et al., 2017).

Así, el estudio de sus predictores en la primera infancia aportará información sobre aspectos importantes para promover de manera temprana, a la vez que nutrirá el desarrollo de políticas públicas especializadas en el tema. Por último, cabe mencionar que, como una primera hipótesis, se espera encontrar diferencias en el desarrollo socioemocional de los niños al año de edad según el tipo de parto realizado, donde los infantes nacidos por cesárea presenten un desarrollo socioemocional más bajo que aquellos nacidos por parto vaginal; ello especialmente en el caso de las cesáreas no programadas, donde la evidencia existente ha demostrado que este procedimiento puede afectar negativamente los cuidados de la madre hacia el niño en el periodo del postparto (Lobel \& DeLuca, 2007). Además, como segunda hipótesis se espera que los niños con madres con mayor sensibilidad cuenten con un mejor desarrollo socioemocional.

La relevancia del presente estudio radica en el impacto que tendría identificar si los factores de tipo de parto y sensibilidad materna permiten predecir un mayor desarrollo socioemocional en los niños de 12 meses, especialmente en el caso del tipo de parto, ya que es un factor poco estudiado en su relación con el desarrollo socioemocional infantil. Asimismo, dar cuenta de una relación entre las variables estudiadas podría generar un efecto en las políticas públicas de salud, además de promover la creación de programas enfocados en el fomento del desarrollo socioemocional durante el primer año de vida en los que se informe y ayude a las madres a desarrollar su sensibilidad — esto soportado en que distintas investigaciones han demostrado 
su efectividad en la intervención positiva de la sensibilidad materna (véase, p. ej., el meta-análisis de BakermansKranenburg et al., 2003) - . Por último, el estudio resulta novedoso debido a que aborda una temática que no ha sido previamente estudiada en el país, y porque aporta una mirada crítica sobre la relación entre el tipo de parto, la sensibilidad materna y el desarrollo socioemocional de los niños a los 12 meses de edad.

\section{Método}

\section{Diseño}

En el presente estudio se implementó un diseño descriptivo, comparativo, correlacional y transversal, con metodología cuantitativa. Específicamente, se describe el desarrollo socioemocional de los niños del estudio al año de edad, se compara el desarrollo socioemocional de los niños según tipo de parto, se correlaciona la sensibilidad de las madres con el desarrollo socioemocional de los niños, y, por último, se analiza la mediación que ejerce la variable sensibilidad en la relación entre el tipo de parto y el desarrollo socioemocional de los niños.

\section{Participantes}

La muestra, no probabilística e intencionada, se compuso de 91 diadas materno filiales seleccionadas a partir de una base mayor que hace parte del proyecto FONDECYT n. ${ }^{\circ} 1160110$, titulado "Mentalización de apoderados y personal educativo y su relación con competencias socioemocionales y lingüísticas de niños(as) de 12 y 30 meses que asisten a sala cuna", en el cual este estudio estuvo inserto.

En particular, el promedio de edad de las madres fue de 27.78 años $(D E=6.7)$, mientras que la edad de los infantes se ubicó en un rango de los 10 a los 15 meses de edad, con un promedio de 12 meses $(D E=1.37)$, siendo un $44.0 \%$ de sexo femenino y un $56.0 \%$ de sexo masculino. Por otra parte, es importante mencionar que la totalidad de los infantes asistía a 27 salas cuna estatales y privadas de 18 comunas de la ciudad de Santiago, Chile; que el 52.74\% de las diadas pertenecía a un NSE bajo, el $24.17 \%$ a un NSE medio, y el $23.07 \%$ a un NSE alto; y que, respecto al nivel educacional de las madres, el $1.8 \%$ contaba con educación básica incompleta, el 2.7 \% tenía educación básica completa, el $11.8 \%$ educación secundaria incompleta, el $27.3 \%$ educación secundaria completa, el $12.7 \%$ tenía formación técnica o universitaria incompleta, el 19.1\% formación técnica o universitaria completa, y el $7.3 \%$ contaba con formación de postgrado.
Finalmente, los requisitos de inclusión para este estudio fueron que las madres tuvieran infantes en un rango de edad entre 10 y 15 meses, que las madres vivieran con su hijo, y que los niños asistieran a jardín infantil o sala cuna. Se excluyeron del estudio aquellos casos en los que el niño o su madre tuvieran alguna psicopatología grave.

\section{Instrumentos}

\section{Cuestionario sociodemográfico.}

Se hizo uso del cuestionario sociodemográfico desarrollado por el equipo principal del cual el presente estudio hace parte. Se trata de un instrumento destinado a caracterizar la muestra, que debe ser contestado por la madre, y que recoge aspectos sociodemográficos del infante - como su edad o sexo-y de sus padres - como el nivel educacional de la madre y el NSE de su familia-; así como información sobre el tipo de parto - vaginal, cesárea o cesárea no programada-.

Escala de sensibilidad del adulto (ESA).

La ESA, desarrollada por Santelices et al. (2012), evalúa en una situación filmada de cinco minutos de juego libre la sensibilidad de adultos significativos en su interacción con niños de 6 a 36 meses de edad. En este ejercicio, la interacción es filmada y luego observada y codificada por un equipo de codificadores previamente entrenados.

Esta escala cuenta con 19 ítems con puntajes entre 1 y 3 puntos - donde un mayor puntaje indica mayor sensibilidad - que se organizan en tres subescalas: respuesta empática, interacción lúdica y expresión emocional. Después de la codificación, los puntajes se promedian para obtener puntajes en un rango entre 1 y 3 para cada subescala, así como para la escala total, a partir de lo cual se alcanzan las categorías de sensibilidad baja, adecuada y alta (Santelices et al., 2012).

Además, el instrumento presenta una adecuada confiabilidad, medida a través del coeficiente alfa de Cronbach $(\alpha=.93)$, y una consistencia interjueces considerable, de .62 (Kappa de Cohen), en una muestra de características similares a la del presente estudio (Santelices et al., 2012). Para la muestra de esta investigación se obtuvo una confiabilidad de $\alpha=.87$.

Functional emotional assessment scale (FEAS).

La FEAS, de Greenspan et al. (2001), evalúa el funcionamiento emocional en infantes de 5 meses a 4 años a través de la observación de su conducta. Específicamente, consiste en una situación filmada del juego libre de 15 a 20 minutos con el investigador, la cual es posteriormente 
codificada a partir de las conductas del cuidador y del niño. Para este estudio, la escala se aplicó a interacciones de juego libre de cinco minutos de duración entre la madre y el niño, y se codificaron solo los ítems correspondientes al niño.

Este instrumento tiene varias formas de aplicación según la edad del niño, de modo que, para el presente estudio, se utilizaron dos formas, una para niños de 10-12 meses y otra para niños de 13-18 meses, considerando tres dimensiones del desarrollo emocional: (a) regulación emocional e interés en el mundo (9 ítems), (b) formación de relaciones o vínculo (5 ítems), y (c) comunicación intencional de doble vía (4 ítems). Cada ítem se puntúa entre 0 y 2 puntos, donde un mayor puntaje indica un mejor funcionamiento.

La confiabilidad de la escala para evaluar las conductas del niño en la muestra original de participantes norteamericanos (Greenspan et al., 2001) fue de $\alpha=.91$ a $\alpha=.97$, con un acuerdo interjueces en un rango entre .91 y .98 (medida con alfa de Cronbach), y de $\alpha=.85$ y $\alpha=.83$ en su adaptación chilena para la forma de 10-12 meses y de 13-18 meses, respectivamente (Gómez, 2019). Para la muestra de este estudio la confiabilidad fue de $\alpha=.80$, con un acuerdo interjueces considerable — de .68 a .72 (Kappa de Cohen)-.

\section{Procedimiento}

La presente investigación consiste en un análisis de datos secundarios de un estudio longitudinal (FONDEYT n. ${ }^{\circ} 1160110$ ), cuyo objetivo fue analizar la influencia de las competencias y características parentales sobre el desarrollo infantil entre los 12 y 30 meses de edad. En dicho trabajo, para la recolección de los datos se estableció contacto con salas cunas para obtener la autorización para aplicar el estudio, se contactó a las madres, y se solicitó la firma de una carta de consentimiento informado a aquellas que estuvieron dispuestas a participar. A continuación, se les pidió responder el cuestionario sociodemográfico y se les invitó a jugar con sus hijos, utilizando un set estándar de juguetes en una habitación de la sala cuna acondicionada para tal fin. Los primeros dos minutos de juego no fueron grabados, con el fin de que se familiarizaran con la evaluación. Posteriormente, se grabaron los cinco minutos de juego libre, y con dicha grabación se codificó la sensibilidad de la madre y el desarrollo socioemocional del niño.

\section{Consideraciones éticas}

El estudio longitudinal del cual se obtuvieron los datos fue revisado y aprobado por el Comité Ético Científico de Ciencias Sociales, Artes y Humanidades de la Pontificia Universidad Católica de Chile (ID: 150707003), y fue llevado a cabo a partir de los estándares éticos de la Declaración de Helsinki de 1964 y sus posteriores enmiendas. En este sentido, las madres firmaron una carta de consentimiento informado al principio del estudio, y los casos fueron identificados con un número de folio para resguardar la confidencialidad de la información.

\section{Análisis de datos}

Se hizo uso del programa R Studio para el análisis de los datos. Para dicho procedimiento, primero se emplearon análisis estadísticos descriptivos de las variables del estudio, considerando: (a) el tipo de parto, con las categorías de parto vaginal, cesárea programada y cesárea no programada; (b) la sensibilidad materna, con las categorías de sensibilidad materna baja, adecuada y alta, y los puntajes para sensibilidad total y subescalas de responsividad, interacción lúdica y sintonía; y (c) el desarrollo socioemocional, con puntajes para escala total, así como para las dimensiones de regulación emocional, vínculos y comunicación. A continuación, se prosiguió con la comprobación de los supuestos de normalidad (prueba Kolmogorov-Smirnov) y homocedasticidad de los datos (prueba de Levene), para luego analizar las diferencias en el desarrollo socioemocional infantil según tipo de parto, primero con un ANOVA, y luego un ANCOVA, controlando el nivel educacional de la madre - al ser una variable que correlacionaba tanto con la sensibilidad materna $(r=.46, p<.001)$ como con el desarrollo socioemocional $(r=.30, p=.004)$ —. Después, se abordó la relación entre la sensibilidad materna y el desarrollo socioemocional infantil a través de correlaciones lineales de Pearson y de correlaciones parciales, controlando el nivel educacional de la madre; y, por último, se realizó una regresión jerárquica con las variables del estudio para determinar un modelo explicativo del desarrollo socioemocional infantil al año de edad.

\section{Resultados}

\section{Análisis descriptivo de las variables de estudio}

Con respecto a las variables de la madre, en el tipo de parto el $59.3 \%$ tuvo un parto vaginal, mientras que el $40.7 \%$ tuvo un parto por cesárea ( $24.2 \%$ programada, $16.5 \%$ no programada); y en cuanto a la sensibilidad materna, el $23.1 \%$ se ubicó en la categoría de baja sensibilidad, mientras que el $59.3 \%$ lo hizo en una sensibilidad adecuada, y el $17.6 \%$ restante en la categoría de sensibilidad alta. La media del puntaje total de la escala fue de $2.08(D E=0.39$, rango $=1.37$ 2.79). La información detallada sobre los descriptivos según las escalas específicas se encuentra en la Tabla 1. 
Tabla 1.

Estadisticos descriptivos para las escalas de sensibilidad materna y desarrollo socioemocional infantil

\begin{tabular}{|c|c|c|c|c|}
\hline & Mín. & Máx. & $M$ & $D E$ \\
\hline \multicolumn{5}{|l|}{ Sensibilidad materna } \\
\hline Responsividad & 1.00 & 3.00 & 1.93 & 0.54 \\
\hline Interacción lúdica & 1.00 & 3.00 & 2.13 & 0.57 \\
\hline Sintonía & 1.43 & 3.00 & 2.22 & 0.42 \\
\hline Puntaje total sensibilidad & 1.37 & 2.79 & 2.08 & 0.39 \\
\hline \multicolumn{5}{|l|}{ Desarrollo socioemocional } \\
\hline Dimensión regulación emocional & 5.6 & 14.0 & 12.22 & 1.86 \\
\hline Dimensión vínculos & 2.0 & 10.0 & 7.98 & 1.93 \\
\hline Dimensión comunicación & 3.0 & 8.0 & 6.09 & 1.49 \\
\hline Puntaje total socioemocional & 10.6 & 32.0 & 26.28 & 4.56 \\
\hline
\end{tabular}

Nota. $\mathrm{N}=91$.

Por otra parte, respecto al desarrollo socioemocional infantil, la media del puntaje total fue de $26.28(D E=4.56$, rango $=10.6-32.0)$ (véase Tabla 1$) ; \mathrm{y}$, aunque no se cuenta con normas nacionales de interpretación de los puntajes, cabe mencionar que el $17.6 \%$ de los niños se ubicó a -1 desviación estándar de la media del grupo.

\section{Análisis comparativo del desarrollo socioemocional infantil} según el tipo de parto

Los resultados de la prueba de Kolmogorov-Smirnov no mostraron diferencias significativas entre estas dos variables $(p>.05)$, de modo que es posible afirmar una distribución normal en el desarrollo socioemocional de los niños según los tres grupos de tipos de parto. De igual forma, la prueba de Levene no fue significativa $(p>.05)$, por lo que se puede evidenciar la igualdad de varianza en los grupos estudiados.
Por otra parte, no se encontraron diferencias en el desarrollo socioemocional de los niños según el tipo de parto, ni en los análisis iniciales $\left(F_{(2,88)}=0.32, p=.728\right)$, ni al controlar el nivel educacional materno $\left(F_{(2,87)}=0.43, p=.655\right)$. No obstante, se observó una tendencia no significativa en la cual los puntajes de desarrollo socioemocional disminuían según el tipo de parto, donde los niños nacidos por parto vaginal tendían a obtener puntajes más altos que aquellos nacidos por cesáreas no programadas (véase Figura 1).

\section{Relación entre sensibilidad materna y desarrollo socioemo- cional infantil}

Los análisis realizados mostraron una correlación significativa entre el puntaje total del desarrollo socioemocional infantil y la sensibilidad materna, tanto en el puntaje total $(r=.43, p<.001)$ como en las subescalas de responsividad $(r=.28, p=.008)$ y sintonía $(r=.48, p<.001)$, por

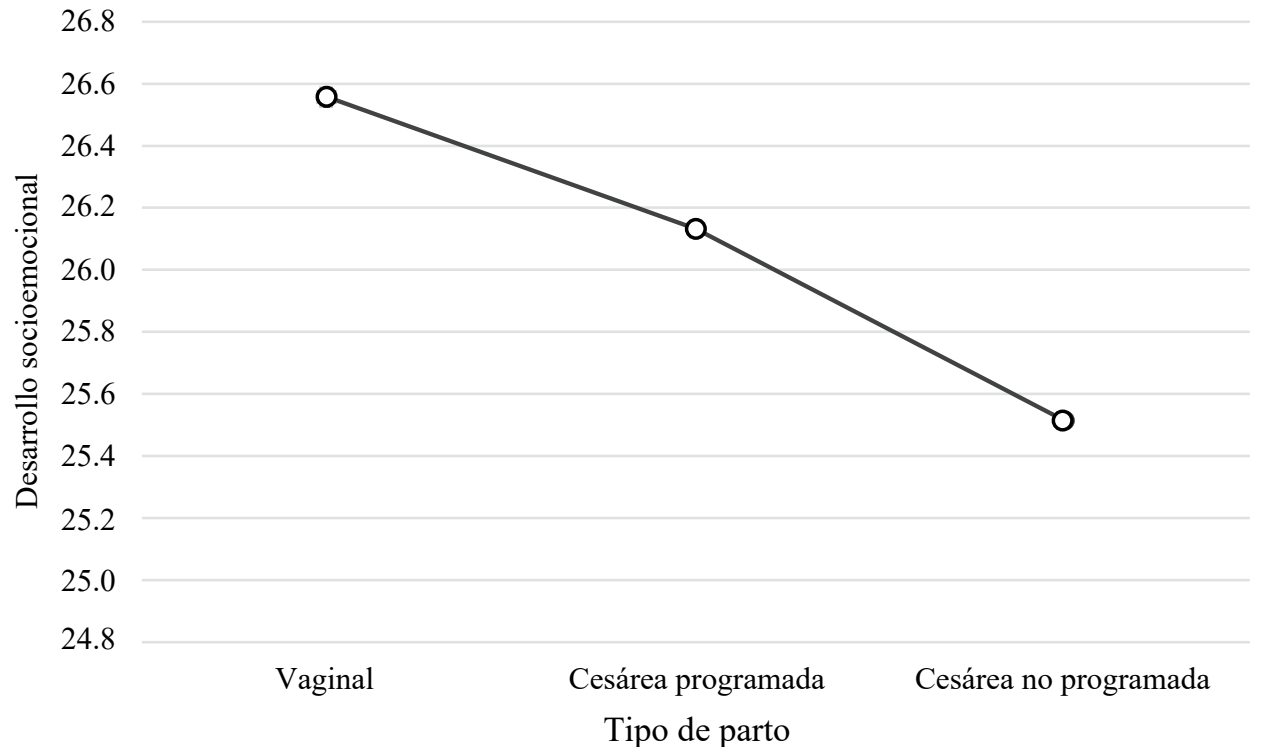

Figura 1. Puntajes en desarrollo socioemocional según el tipo de parto. 
lo que se encuentra que a mayor sensibilidad de la madre, mayor desarrollo socioemocional del niño. Esto mismos resultados se aprecian para las dimensiones de desarrollo socioemocional (véase Tabla 2). La escala de interacción lúdica en sensibilidad fue la única que no correlacionó con el desarrollo socioemocional infantil.

Por otra parte, al repetir los análisis controlando el nivel educacional materno, se encontró que se mantienen las correlaciones significativas para la escala total de sensibilidad, con correlaciones moderadas para la escala total de desarrollo socioemocional $(r=.33, p<.001)$ y para la dimensión de regulación $(r=.30, p=.004)$, mientras que las dimensiones de vínculos $(r=.28, p=.007)$ y comunicación $(r=.27$, $p=.011)$ presentaron correlaciones débiles. Además, se observaron correlaciones significativas entre la escala de sintonía de sensibilidad, con correlaciones moderadas para la escala total de desarrollo socioemocional $(r=.40$, $p<.001)$ y para las dimensiones de regulación $(r=.39$, $p<.001)$, vínculos $(r=.30, p=.004)$ y comunicación $(r=.33, p=.002)$.

Modelo explicativo del desarrollo socioemocional infantil al año de edad

Por último, se llevó a cabo una regresión jerárquica para obtener un modelo explicativo del desarrollo socioemocional a esta edad - tomando en cuenta el puntaje total para esta variable - . Para ello, en un primer momento se analizaron las correlaciones entre el desarrollo socioemocional de los niños y las variables de sexo y edad del niño, y de edad y educación de la madre, para decidir las variables a incluir en el modelo. De estos análisis, solo resultaron ser significativos la edad del niño $(r=-.27, p=.011)$ y el nivel educacional de la madre $(r=.30, p=.004)$, por lo cual fueron estas las variables que se consideraron para el análisis. Por esta misma razón se consideró el puntaje total de sensibilidad de las madres, pero no así el tipo de parto, ya que análisis previos no mostraron relación con la variable en estudio. En conclusión, en el análisis se introdujeron como variables de control la edad de los niños (Modelo 1), el nivel educacional materno (Modelo 2), y, por último, el puntaje total en sensibilidad materna (Modelo 3 ) (véase Tabla 3).

Con este análisis, en el Modelo 1 se obtuvo que las diferencias con respecto a la edad del niño son significativas $(\beta=-.27, t=-2.59, p=.011)$, pues, a mayor edad, menor puntaje en desarrollo socioemocional - con un $7.0 \%$ de la varianza del constructo explicada- Esta variable se mantuvo significativa hasta el modelo final. Por otra parte, el nivel educacional de la madre (Modelo 2) también resultó ser un predictor significativo del desarrollo socioemocional $(\beta=.34, t=3.46, p<.001)$ cuando los valores de edad del niño se mantenían fijos; así, un mayor nivel educacional de la madre se relacionó con un mayor puntaje en el desarrollo socioemocional del niño —aportando un $11.1 \%$ adicional en la explicación de la varianza del constructo-. No obstante, en el modelo final esta variable dejó de ser significativa.

Finalmente, la sensibilidad materna total también fue un predictor significativo $(\beta=.37, t=3.46, p<.001)$ cuando se introdujo en el Modelo 3, habiendo controlado la edad del niño y la educación de la madre. Ello indica que, como se esperaba, una mayor sensibilidad materna se relaciona con un mayor puntaje en el desarrollo socioemocional del niño. De hecho, la sensibilidad materna aportó un $10.0 \%$ adicional en la explicación de la varianza del constructo y fue el predictor más significativo en el modelo final, seguido por la edad del niño. Así, el modelo final (Modelo 3) resultó ser significativo $\left(F_{(3,87)=} 11.31, p<.001\right)$, dando cuenta de un $28.0 \%$ de la varianza total del desarrollo socioemocional.

Por último, se repitieron los análisis de regresión con las distintas escalas de sensibilidad con el fin de detectar cuál de ellas resultaba ser más significativa para el desarrollo socioemocional infantil, a partir de lo cual se encontró que la subescala de sintonía resultó ser el predictor más

Tabla 2.

Correlaciones entre sensibilidad materna y desarrollo socioemocional infantil

\begin{tabular}{|c|c|c|c|c|c|}
\hline \multirow{2}{*}{ Sensibilidad } & & \multicolumn{4}{|c|}{ Escala socioemocional } \\
\hline & & Puntaje total & Autorregulación & Vínculos & Comunicación \\
\hline \multirow{2}{*}{ Puntaje total } & Corr. & .43 & .34 & .40 & .35 \\
\hline & Sig. & .000 & .001 & .000 & .001 \\
\hline \multirow{2}{*}{ Responsividad } & Corr. & .28 & .20 & .29 & .23 \\
\hline & Sig. & .008 & .063 & .006 & .029 \\
\hline \multirow{2}{*}{ Interacción lúdica } & Corr. & .20 & .15 & .19 & .17 \\
\hline & Sig. & .060 & .147 & .074 & .106 \\
\hline \multirow{2}{*}{ Sintonía } & Corr. & .48 & .41 & .42 & .41 \\
\hline & Sig. & .000 & .000 & .000 & .000 \\
\hline
\end{tabular}

Nota. $\mathrm{N}=91$. 
Tabla 3.

Modelo predictivo del desarrollo socioemocional infantil

\begin{tabular}{|c|c|c|c|c|c|c|c|c|c|c|c|c|}
\hline \multirow{2}{*}{ Variables } & \multicolumn{3}{|c|}{ Modelo 1} & \multicolumn{3}{|c|}{ Modelo 2} & \multicolumn{3}{|c|}{ Modelo 3} & \multicolumn{3}{|c|}{ Modelo 3b } \\
\hline & $B$ & $S E$ & $\beta$ & $B$ & $S E$ & $\beta$ & $B$ & $S E$ & $\beta$ & $B$ & $S E$ & $\beta$ \\
\hline Constante & 36.90 & 4.12 & & 33.69 & 4.00 & & 27.57 & 4.17 & & 25.65 & 4.24 & \\
\hline Edad del niño & -0.89 & 0.34 & $-.27 * *$ & -1.03 & 0.33 & $-.31 * *$ & -1.03 & 0.31 & $-.31 * *$ & -0.93 & 0.30 & $-.28 * *$ \\
\hline Educación de la madre & & & & 1.09 & 0.32 & $.34 * *$ & 0.44 & 0.35 & .14 & 0.36 & 0.35 & .11 \\
\hline Sensibilidad & & & & & & & 4.36 & 1.26 & $.37 * *$ & & & \\
\hline Subescala de sintonía & & & & & & & & & & 4.55 & 1.16 & $.42 * *$ \\
\hline $\mathrm{r}^{2}$ & & .070 & & & $.182 * *$ & & & $.280 * *$ & & & $.304 * *$ & \\
\hline $\mathrm{F}$ & & 6.7 & & & 9.76 & & & 11.31 & & & 12.69 & \\
\hline
\end{tabular}

Nota. $* * p<.01$.

significativo $(\beta=.42, t=3.92, p<.001)$ (véase Tabla 3, Modelo 3b), dando cuenta de un $12.3 \%$ de la varianza, y habiendo controlado la edad del niño y la educación de la madre. Este modelo también resultó ser significativo $\left(F_{(3,87)}=12.69, p<.001\right)$, dando cuenta de un $30.4 \%$ de la varianza total del constructo.

\section{Discusión}

Tal como se había planteado en las hipótesis iniciales, los resultados del estudio respaldan una relación entre una mayor sensibilidad materna y un mejor desarrollo socioemocional de los niños al año de edad, además de que la sensibilidad es el predictor más importante para este desarrollo - en especial la dimensión de sintonía de este constructo-, en comparación con las otras variables estudiadas; resultado coherente con lo encontrado en otros estudios donde se demuestra la relevancia de la sensibilidad materna para el desarrollo general del niño en aspectos tanto cognitivos como emocionales, psicomotores, sociales y lingüísticos (Simó \& D’Ocon, 2014). Si bien la relación entre estas dos variables ya había sido documentada en otros países (Chen, 2012; Page et al., 2010), esta misma no había sido estudiada en una muestra chilena ni con niños de esta edad, de modo que la presente investigación aporta evidencia nacional sobre la importancia de la sensibilidad materna en el desarrollo socioemocional infantil.

En este sentido, teniendo en cuenta que casi el $18 \%$ de los niños ya se encontraba bajo la media en su desarrollo socioemocional en comparación a su grupo, y que el $23 \%$ de las madres presentaba una sensibilidad baja, resulta de gran relevancia el hecho de promover tempranamente una sensibilidad adecuada en las madres, lo cual, como se demuestra en el estudio, beneficiaría el desarrollo socioemocional de los niños. Sin embargo, debe considerarse que este es un estudio con una muestra pequeña y no representativa de todos los niños chilenos, por lo cual sus resultados deben tomarse con cautela; aunque con estos hallazgos es posible sugerir, por ejemplo, que se incluya la detección y promoción de competencias maternas adecuadas en los controles de salud que se le hacen al niño durante el primer año de vida, lo cual contribuiría, en parte, a favorecer su óptimo desarrollo socioemocional.

Por otra parte, no se apreciaron diferencias significativas respecto a la comparación del desarrollo socioemocional de los niños según el tipo de parto, aunque sí se observó una tendencia no significativa a que los puntajes totales de desarrollo socioemocional disminuyeran cuando el parto era por cesárea. En este punto, considerando que en casi el $41 \%$ de los casos el parto fue por cesárea - programada o no-, un resultado bastante superior al 10-15\% recomendado por la OMS (WHO, 1985, citado por Salinas et al., 2007), sería relevante realizar nuevos estudios sobre el posible impacto del tipo de parto sobre el desarrollo socioemocional de los niños, en los cuales se considere una muestra de mayor tamaño y niños de otros rangos etarios.

De hecho, en estudios previos se ha constatado la influencia del tipo de parto en relación con el comportamiento de la madre frente al bebé, pues se ha encontrado en madres con este tipo de parto un humor depresivo y ansiedad (Lobel \& DeLuca, 2007; Ryding et al., 1998; Uriarte, 2006), comportamientos que impactan negativamente sobre el vínculo entre la madre y su hijo (Van Reenen \& Van Rensburg, 2013), lo que, a su vez, podría repercutir en su desarrollo socioemocional. En relación con esto, en algunos estudios se han identificado mayores problemas socioemocionales y conductuales en niños cuyas madres presentaron síntomas depresivos o ansiosos antes o después del parto (Madigan et al., 2018; Raskin et al., 2016); y en otros se ha encontrado que altos niveles de ansiedad materna en etapas tempranas del embarazo predicen posteriormente una mayor negatividad y rechazo, así como peores estrategias de regulación en los niños antes del año de edad — relación que, a su 
vez, es moderada por la sensibilidad materna- (Thomas et al., 2017). Así, futuros estudios podrían incluir la salud mental de la madre durante el embarazo, o tras el parto, de modo que establezcan su relación con el desarrollo socioemocional del niño.

Además, cabe considerar que los estudios realizados sobre este tema han sido desarrollados durante los primeros meses de vida del niño, por lo que faltan estudios que aborden si las repercusiones del parto serían solo a corto plazo, o si tendrían un efecto a largo plazo. Al ser este un estudio con una muestra muy acotada, la falta de resultados significativos no permite responder la pregunta y nuevos estudios son necesarios. Incluso, cabría considerar que el hecho de que todos los niños asistieran a sala cuna podría dar cuenta de un factor de apoyo externo al hogar, lo que homogeneizaría el nivel de desarrollo socioemocional de los niños. Por ello, futuros estudios deberían incluir niños que no asisten a sala cuna, sobre todo porque solo un $29.1 \%$ de los niños de 0 a 3 años asiste a sala cuna en Chile (Ministerio de Desarrollo Social, 2018).

Adicional a esto, se han descrito algunos factores que afectarían la forma en que cada madre experiencia su tipo de parto, como lo serían las creencias y expectativas previas, así como la percepción del apoyo necesitado y recibido por sus parejas o por las matronas que las acompañan durante el proceso (Ip et al., 2003). En este sentido, futuros estudios podrían incluir la interacción del tipo de parto con otros elementos que consideren la forma en que este fue vivenciado, donde, por ejemplo, una vivencia del parto como una experiencia traumática y de poco apoyo podría tener un impacto mayor sobre el vínculo con el hijo.

Finalmente, es importante tener en cuenta algunas limitaciones del presente estudio, como el hecho de que la muestra haya sido no probabilística y de un tamaño reducido (91 diadas); que todos los niños de la muestra asistían a sala cuna, pública o privada - de modo que los resultados no podrían ser generalizados a todos los niños de 12 meses-; o que la evaluación del desarrollo socioemocional de los niños se realizó con base en la observación de videos de cinco minutos de interacción por lo que cabe la pregunta de si observaciones de mayor duración generarían resultados distintos en los niños-. Así, los resultados encontrados deben ser tomados con cautela, y profundizarse con futuros estudios. Por otra parte, al ser este un estudio transversal, los resultados únicamente da cuenta de lo que ocurre en estas variables para estos niños al año de edad, de manera que sería importante investigar en estudios futuros la relación entre estas variables en niños de otras edades, y considerando un seguimiento longitudinal.

Además, al incluir una muestra de mayor tamaño y más representativa, e incluyendo niños de distintas edades, futuros estudios en Chile podrían indagar si el tipo de parto tiene repercusiones en otros aspectos del desarrollo infantil, por ejemplo, en el desarrollo cognitivo o lingüístico. Incluso, otros aspectos que podrían tomarse en cuenta para analizar su efecto sobre el desarrollo socioemocional infantil serían los relacionados con el bienestar psicológico de la madre, como lo sería la depresión postparto, que afecta a una de cada ocho mujeres y se ha asociado a efectos negativos tanto en la madre como en el desarrollo físico, cognitivo y emocional de los infantes (Patel et al., 2012).

Asimismo, futuros estudios podrían considerar si la sensibilidad materna es condicional a la edad del niño — como se encontró en este estudio con respecto al desarrollo socioemocional-, controlando el tipo de parto. Ello permitiría analizar si la sensibilidad materna va cambiando conforme el niño crece, si esta trayectoria de cambio es diferente para niños nacidos en partos por cesárea o vaginales, o si, en caso contrario, los efectos del tipo de parto asociados a la sensibilidad de la madre se presenten solo en los primeros meses, mientras que a largo plazo pueden desaparecer.

Pese a estas limitaciones, este estudio aporta evidencias sobre la relevancia de promover la sensibilidad materna durante el primer año de vida del bebé, aspecto que debería ser considerado en el diseño de planes de apoyo a la infancia temprana. Una detección oportuna, así como su promoción y prevención, permitirán apoyar desde un inicio el desarrollo socioemocional de los infantes, preparándolos de una mejor manera para los desafíos del futuro.

\section{Referencias}

Ainsworth, M., Blehar, M., Waters, E., \& Wall, S. (1978). Patterns of attachment: a psychological study of the strange situation. Lawrence Erlbaum Associates.

Atkinson, L., Niccols, A., Paglia, A., Coolbear, J., Parker, K. C., Poulton, L., Guger, S., \& Sitarenios, G. (2000). A meta-analysis of time between maternal sensitivity and attachment assessments: implications for internal working models in Infancy/toddlerhood. Journal of Social and Personal Relationships, 17(6), 791-810. https://doi. org/10.1177/0265407500176005

Bakermans-Kranenburg, M. J., Van Ijzendoorn, M. H., \& Juffer, F. (2003). Less is more: meta-analyses of sensitivity and attachment interventions in early childhood. Psychological Bulletin, 129(2), 195-215. https://doi. org/10.1037/0033-2909.129.2.195

Betrán, A. P., Merialdi, M., Lauer, J. A., Bing-Shun, W., Thomas, J., Van Look, P., \& Wagner, M. (2007). Rates of caesarean section: analysis of global, regional and national estimates. Paediatric and Perinatal Epidemiology, 21(2), 98-113. https://doi.org/10.1111/j.1365-3016.2007.00786.x 
Berger, C., Milicic, N., Alcalay, L., Torretti, A., Arab, M., \& Justiniano, B. (2009). Bienestar socio-emocional en contextos escolares: la percepción de estudiantes chilenos. Estudios sobre Educación, 17, 21-43. https://dadun.unav. edu/bitstream/10171/9839/3/17Eb.pdf

Bernier, A., Jarry-Boileau, V., Tarabulsy, G. M., \& Miljkovitch, R. (2010). Initiating a caregiving relationship: Pregnancy and childbirth factors as predictors of maternal sensitivity. Infancy, 15(2), 197-208. https://doi. org/10.1111/j.1532-7078.2009.00006.x

Bordoni, M. (2018). El entonamiento afectivo en las interacciones tempranas adulto-bebé: una revisión. Revista Colombiana de Psicología, 27(1), 13-25. https://doi. org/10.15446/rcp.v27n1.61019

Bornstein, M. H., \& Putnick, D. L. (2012). Cognitive and socioemotional caregiving in developing countries. Child Development, 83(1), 46-61. https://doi. org/10.1111/j.1467-8624.2011.01673.x

Bornstein, M. H., Hendricks, C., Haynes, O. M., \& Painter, K. (2007). Maternal sensitivity and child responsiveness: associations with social context, maternal characteristics, and child characteristics in a multivariate analysis. Infancy, 12(2), 189-223. https://doi.org/10.1111/j.1532-7078.2007. tb00240.x

Bowlby, J. (1969). El apego. Paidós.

Chen, X. (2012). Culture, peer interaction, and socioemotional development. Child Development Perspectives, 6, 27-34. https://doi.org/10.1111/j.1750-8606.2011.00187.x

De Andrés, C. (2005). La educación emocional en edades tempranas y el interés de su aplicación en la escuela. Programas de educación emocional, nuevo reto en la formación de los profesores. Tendencias Pedagógicas, 10, 107-124. https://repositorio.uam.es/bitstream/hand le/10486/4739/31241_2005_10_05.pdf

Doesum, K., Hosman, C., Riksen-Walraven, M., \& Hoefnagles, C. (2007). Correlates of depressed mothers' sensitivity toward their infants: the role of maternal, child, and contextual characteristics. Journal of the American Academy of Child \& Adolescent Psychiatry, 46(6), 747-756. https://doi. org/10.1097/CHI.0b013e318040b272

Domitrovich, C. E., Durlak, J. A., Staley, K. C., \& Weissberg, R. P. (2017). Social-emotional competence: An essential factor for promoting positive adjustment and reducing risk in school children. Child Development, 88(2), 408-416. https://doi.org/10.1111/cdev.12739

Farkas, C., Vallotton, C. D., Strasser, K., Santelices, M. P., \& Himmel, E. (2017). Socioemotional skills between 12 and 30 months of age on Chilean children: when do the competences of adults matter? Infant Behavior and Development, 49, 192-203. https://doi.org/10.1016/j.infbeh.2017.09.010

Gálvez, A., \& Farkas, C. (2017). Relación entre mentalización y sensibilidad de madres de infantes de un año de edad y su efecto en su desarrollo socioemocional. Psykhe, 26(1), 1-14. https://doi.org/10.7764/psykhe.26.1.879
Field, T. (2010). Postpartum depression effects on early interactions, parenting, and safety practices: a review. Infant Behavior and Development, 33(1), 1-6. https://doi. org/10.1016/j.infbeh.2009.10.005

Goker, A., Yanikkerem, E., Demet, M. M., Dikayak, S., Yildirim, Y., \& Koyuncu, F. M. (2012). Postpartum depression: is mode of delivery a risk factor?. ISRN Obstetrics and Gynecology, 2012, 1-6. https://doi.org/10.5402/2012/616759

Gómez, D. (2019). Estudio longitudinal de la relación entre las interacciones parentales y el desarrollo de habilidades socioemocionales en niños a los 12 y a los 30 meses de edad, considerando el temperamento del niño (tesis de doctorado no publicada). Pontificia Universidad Católica de Chile, Santiago, Chile.

Greenspan, S., DeGandi, G., \& Wieder, S. (2001). The functional emotional assessment scale (FEAS) for infancy and early childhood: clinical and research application. Interdisciplinary council on developmental and learning disorders.

I Font, L. C. (2015). Miedo al parto y narrativas intergeneracionales: Una aproximación desde la antropología. Dilemata, 18, 129-145. https://www.dilemata.net/revista/index.php/ dilemata/article/view/376

Ip, W. Y., Chien, W. T., \& Chan, C. L. (2003). Childbirth expectations of Chinese first-time pregnant women. Journal of Advanced Nursing, 42(2), 151-158. https://doi. org/10.1046/j.1365-2648.2003.02598.x

Kemppinen, K., Kumpulainen, K., Moilanen, I., \& Ebeling, H. (2006). Recurrent and transient depressive symptoms around delivery and maternal sensitivity. Nordic Journal of Psychiatry, 60(3), 191-199. https://doi. org/10.1080/08039480600635975

Kiss, M., Fechete, G., Pop, M., \& Susa, G. (2014). Early childhood self-regulation in context: Parental and familial environmental influences. Cognition, Brain, Behavior, 18(1), 55-85. https://www.researchgate.net/publica tion/279954552_Early_childhood_self-regulation_in_con text_Parental_and_familial_environmental_influences

Kopystynska, O., Spinrad, T. L., Seay, D. M., \& Eisenberg, N. (2016). The interplay of maternal sensitivity and gentle control when predicting children's subsequent academic functioning: Evidence of mediation by effortful control. Developmental Psychology, 52(6), 909-921. https://doi. org $/ 10.1037 / \mathrm{dev} 0000122$

Lee, A., \& Kirkham, M. (2008). Disciplinary discourses: rates of caesarean section explained by medicine, midwifery and feminism. Health Care for Women International, 29(5), 448-467. https://doi.org/10.1080/07399330801949574

Lecannelier, F., Kimelman, M., González, L., Nuñez, C., \& Hoffmann, M. (2008). Evaluación de patrones de apego en infantes durante su segundo año en dos centros de atención de Santiago de Chile. Revista Argentina de Clínica Psicológica, 17(3), 197-207. https://www.redalyc.org/ pdf/2819/281921795001.pdf 
Lobel, M., \& DeLuca, R. (2007). Psychosocial sequelae of cesarean delivery: review and analysis of their causes and implications. Social Science \& Medicine, 64(11), 2272-2284. https://doi.org/10.1016/j.socscimed.2007.02.028

Madigan, S., Oatley, H., Racine, N., Fearon, R. P., Schumacher, L., Akbari, E., Cooke, J., \& Tarabulsy, G. M. (2018). A meta-analysis of maternal prenatal depression and anxiety on child socio-emotional development. Journal of the American Academy of Child \& Adolescent Psychiatry, 57(9), 645-657. https://doi.org/10.1016/j.jaac.2018.06.012

Ministerio de Salud. (2015). Guía Perinatal. Santiago. http://www.repositoriodigital.minsal.cl/bitstream/ handle/2015/436/GUIA-PERINATAL_2015-PARAPUBLICAR.pdf?sequence $=1$ \&isAllowed $=\mathrm{y}$

Ministerio de Desarrollo Social. (2018). Informe de Desarrollo Social 2018, Gobierno de Chile. http://www.ministeriode sarrollosocial.gob.cl/storage/docs/Informe_de_Desarrollo_ Social_2018.pdf

Morera, S., Santelices, M. P., \& Farkas, C. (2012). Habilidades parentales requeridas en la interacción con el preescolar para la promoción del apego seguro. Humanitas: Revista de Investigación, 9(9), 168-189. https://dialnet.unirioja.es/ descarga/articulo/4033902.pdf

Moed, A., Dix, T., Anderson, E. R., \& Greene, S. M. (2017). Expressing negative emotions to children: Mothers' aversion sensitivity and children's adjustment. Journal of Family Psychology, 31(2), 224-233. https://doi.org/10.1037/ fam0000239

Osorio, H. D. L., González, I. C., \& Trujillo, L. E. (2018). Afectividad y apoyo social percibido en mujeres gestantes: Un análisis comparativo. Revista Colombiana de Psicología, 27(2), 85-101. https://doi.org/10.15446/rcp. v27n2.65584

Palmer, F. B., Anand, K. J., Graff, J. C., Murphy, L. E., Qu, Y., Völgyi, E., Rovnaghi, C. R., Moore, A., Tran, Q. T., \& Tylavsky, F. A. (2013). Early adversity, socioemotional development, and stress in urban 1-year-old children. The Journal of Pediatrics, 163, 1733-1739. https://doi. org/10.1016/j.jpeds.2013.08.030

Page, M., Wilhelm, M., Gamble, W., \& Card, N. (2010). A comparison of maternal sensitivity and verbal stimulation as unique predictors of infant social-emotional and cognitive development. Infant Behavior and Development, 33, 101110. https://doi.org/10.1016/j.infbeh.2009.12.001

Patel, M., Bailey, R., Jabeen, S., Ali, S., Barker, N., \& Osiezagha, K. (2012). Postpartum depression: A review. Journal of Health Care for the Poor and Underserved, 23(2), 534-42. https://doi.org/10.1353/hpu.2012.0037

Pelchat, D., Bisson, J., Bois, C., \& Saucier, J. F. (2003). The effects of early relational antecedents and other factors on the parental sensitivity of mothers and fathers. Infant and Child Development, 12(1), 27-51. https://doi.org/10.1002/ icd.335
Peters, L. L., Thornton, C., de Jonge, A., Khashan, A., Tracy, M., Downe, S., Feijen-de Jong, E., \& Dahlen, H. G. (2018). The effect of medical and operative birth interventions on child health outcomes in the first 28 days and up to 5 years of age: a linked data population-based cohort study. Birth, 45(4), 347-357. https://doi.org/10.1111/birt.12348

Raskin, M., Easterbrooks, M. A., Lamoreau, R. S., Kotake, C., \& Goldberg, J. (2016). Depression trajectories of antenatally depressed and nondepressed young mothers: implications for child socioemotional development. Women's Health Issues, 26(3), 344-350. https://doi.org/10.1016/j. whi.2016.02.002

Ryding, E. L., Wijma, K., \& Wijma, B. (1998). Psychological impact of emergency cesarean section in comparison with elective cesarean section, instrumental and normal vaginal delivery. Journal of Psychosomatic Obstetrics \& Gynecology, 19(3), 135-144. https://doi. org/10.3109/01674829809025691

Salinas, H., Naranjo, B., Pastén, J., \& Retamales, B. (2007). Estado de la cesárea en Chile. Riesgos y beneficios asociados a esta intervención. Revista HCUCh, 18, 168-78. https://www.redclinica.cl/portals/0/users/014/14/14/publi caciones/revista/estado_cesarea_en_chile.pdf

Santelices, M. P., Carvacho, C., Farkas, C., León, F., Galleguillos, F., \& Himmel, E. (2012). Medición de la sensibilidad del adulto con niños de 6 a 36 meses de edad: construcción y análisis preliminares de la Escala de Sensibilidad del Adulto, ESA. Terapia Psicológica, 30(3), 19-29. https:// doi.org/10.4067/S0718-48082012000300003

Santelices, M. P., Farkas, C., Montoya, M. F., Galleguillos, F., Carvacho, C., Fernández, A., Morales, L., Taboada, C., \& Himmel, E. (2015). Factores predictivos de sensibilidad materna en infancia temprana. Psicoperspectivas, 14(1), 66-76. https://doi.org/10.5027/ psicoperspectivas-vol14-issue1-fulltext-441

Shin, H., Park, Y. J., \& Kim, M. J. (2006). Predictors of maternal sensitivity during the early postpartum period. Journal of Advanced Nursing, 55(4), 425-434. https://doi. org/10.1111/j.1365-2648.2006.03943.x

Simó, S., \& D’Ocon, A. (2014). La estructura temporal de la experiencia de sensibilidad materna: su efecto sobre el desarrollo cognitivo y emocional infantil. Infancia y Aprendizaje, 34(4), 481-493. https://doi. org/10.1174/021037011797898421

Swain, J. E., Tasgin, E., Mayes, L. C., Feldman, R., Todd Constable, R., \& Leckman, J. F. (2008). Maternal brain response to own baby-cry is affected by cesarean section delivery. Journal of Child Psychology and Psychiatry, 49(10), 10421052. https://doi.org/10.1111/j.1469-7610.2008.01963.x

Sword, W., Kurtz Landy, C., Thabane, L., Watt, S., Krueger, P., Farine, D., \& Foster, G. (2011). Is mode of delivery associated with postpartum depression at 6 weeks: a prospective cohort study. BJOG: An International Journal of 
Obstetrics \& Gynaecology, 118(8), 966-977. https://doi. org/10.1111/j.1471-0528.2011.02950.x

Thomas, J. C., Letourneau, N., Campbell, T. S., TomfohrMadsen, L., Giesbrecht, G. F., \& APrON Study Team. (2017). Developmental origins of infant emotion regulation: mediation by temperamental negativity and moderation by maternal sensitivity. Developmental Psychology, 53(4), 611-628. https://doi.org/10.1037/dev0000279

Uriarte, A. G. (2006). Estados emocionales en el postparto. Medicina Naturista, 10, 483-487. https://dialnet.unirioja.es/ servlet/articulo?codigo $=1985554$
Van Reenen, S. L., \& Van Rensburg, E. (2013). The influence of an unplanned caesarean section on initial motherinfant bonding: mothers' subjective experiences. Journal of Psychology in Africa, 23(2), 269-274. https://doi.org/10.10 80/14330237.2013.10820623

Xie, R. H., Lei, J., Wang, S., Xie, H., Walker, M., \& Wen, S. W. (2011). Cesarean section and postpartum depression in a cohort of Chinese women with a high cesarean delivery rate. Journal of Women's Health, 20(12), 1881-1886. https://doi.org/10.1089/jwh.2011.2842 